\title{
Combined General And Epidural Anesthesia Without The Use Of Intravenous Analgesic Medications
}

\author{
Antônio Carlos Meinberg ${ }^{1}$ \\ ${ }^{1}$ Affiliation not available
}

November 30, 2021

Antonio Carlos Meinberg ${ }^{1}$ and Idiberto José Zotarelli Filho ${ }^{2}$

School of Medicine of São José do Rio Preto, Famerp - Discipline of Anesthesiology, São José do Rio Preto, Brazil.

Faceres - College of Medicine, São José do Rio Preto, São Paulo, Brazil.

\section{INTRODUCTION}

Regional anesthesia associated with general anesthesia can lead to the reduction of harmful mediators (catecholamines, endogenous corticosteroids) to the normal physiology of the body [1,2]. Reduced stress hormones are referred to in most studies ever done. Despite this beneficial effect, regional anesthesia can add blood flow reduction to various tissues, as it can lead to sympathectomy due to spinal cord levels blocked by local anesthetics very commonly used. This effect can be accentuated by drugs administered in general anesthesia (inhalation agents and inducers) [3].

In this context, a previous study analyzed the side effects of postoperative epidural analgesia controlled with bupivacaine/sufentanil versus an epidural bolus (BOLUS) of clonidine/morphine in 68 patients with pancreatic surgery. Postoperative pain treatment was carried out for 4 days, with $0.25 \%$ bupivacaine and 2 micrograms/mL sufentanil, adjusted to a $3 \mathrm{~mL}$ bolus and $10 \mathrm{~min}$ blockade time. BOLUS patients received injections of 150 microgram clonidine plus $2 \mathrm{mg}$ morphine on demand. It was concluded that, because of superior analgesia and reduced side effects with similar costs, controlled postoperative epidural analgesia is preferable to the BOLUS technique for the treatment of postoperative pain. The epidural clonidine/morphine bolus technique resulted in inferior analgesia, more side effects, and comparable costs compared to a controlled epidural analgesia regimen after abdominal surgery [4].

In this sense, in confirmation of the scientific evidence from the previous study, a recent systematic review study carried out in 2021 explored the efficacy of different pain relief modalities used in the treatment of postoperative pain after pancreaticoduodenectomy (PD) and distal pancreatectomy (DP) and impact on perioperative outcomes. Five randomized controlled trials and seven retrospective cohort studies $(1,313$ patients) were included in the systematic review. Studies compared epidural analgesia (EDA) (n=845), patient-controlled analgesia $(\mathrm{PCA})(\mathrm{n}=425)$ and transabdominal wound catheters (TAWC) $(\mathrm{n}=43)$. EDA versus PCA after PD was compared in eight studies (1,004 patients) in the quantitative meta-analysis. Pain scores on days $2(\mathrm{p}=0.19)$ and $4(\mathrm{p}=0.18)$ and respiratory morbidity $(\mathrm{p}=0.42)$ were comparable between UDE and PDA. Operative times, bile leakage, delayed gastric emptying, pancreatic fistula, opioid use, and length of stay were also comparable between EDA and PDA. Pain scores and perioperative outcomes were comparable between EDA and PCA after PD and EDA and TAWC after PD [5].

In this sense, regional anesthesia associated with general anesthesia can lead to a reduction of harmful mediators (catecholamines, endogenous corticosteroids) to the normal physiology of the body. Reduced stress 
hormones are referred to in most studies ever done. Despite this beneficial effect, regional anesthesia can add blood flow reduction to various tissues, as this can lead to sympathectomy in spinal levels blocked by very commonly used Local anesthetics. This effect can be accentuated by drugs administered in general anesthesia (inhalation agents and inductors). Because of this finding, I recommended in this study the use of medications that do not produce neuraxial sympathectomy, which can lead to an excellent analgesic status both intraoperatively (CLONIDINE+SUFENTANIL) and postoperatively (CLONIDINE plus MORPHINE).

Because of this finding, the use of medications that do not produce neuraxial sympathectomy will be evaluated in this study, which may lead to an excellent analgesic status both intraoperatively (clonidine and sufentanil) and postoperatively (clonidine and morphine). Therefore, this study aimed to assess hemodynamic variation, safety, immediate postoperative pain during the recommended anesthetic technique, aiming for an anesthetic plan around 50 in the BIS and perfusion parameters: Lactate below 2, mixed venous saturation above $80 \%$, arterial and venous CO2 difference less than six, and hemoglobin above 10g/dL.

\section{METHODS}

\section{Study Design}

This study will follow a prospective observational longitudinal model with individuals who will undergo major surgical procedures. Follow-up time will be six months. The STROBE clinical research rules will be used (Strengthening the Reporting of Observational Studies in Epidemiology, available at: https://www.strobestatement.org/).

\section{Sample Calculation}

More than 100 individuals will be evaluated in the eligibility process, based on the inclusion and exclusion criteria. For sample calculation, a standard deviation of 30, a confidence interval of $95.0 \%$, and a margin of error of $10 \%$ for the confidence interval were estimated, resulting in a sample of 38 individuals.

\section{Ethical Aspects}

This study will be duly presented at the Research Ethics Committee at Faceres, College of Medicine of São José do Rio Preto/SP. Informed consent will be obtained by the principal investigator, based on the 1964 Declaration of Helsinki of the World Medical Association, also following the guidelines of Resolution 466/2012 of the National Health Council-Ministry of Health (Brasil, 2012).

\section{Participants and Interventions}

When the patient arrives in the operating room, he signs the term of adherence to the work protocol and is monitored immediately afterward. He then receives anxiolytic medication (midazolam) until tranquilization. Minutes later, the client sits down for epidural puncture, in T9/T10 to T12/L1 in upper floor surgeries (pancreatectomy, nephrectomy, aortic aneurysm, thoracotomy, and hepatectomy, or L3/L4, L2/L3 for lower floor surgeries of the abdomen (cystectomy, rectosigmoidectomy, prostatectomy. The epidural solution to be administered:

- Clonidine $3 \mathrm{ug} / \mathrm{kg}$

- Sufentanil 30ug

- Morphine $30 \mathrm{ug} / \mathrm{Kg}$

- Thus, the proportion is $3 / 30 / 30$.

The patient then lies down to start general anesthesia with Propofol at a dose up to Bis around 40 and atracurium at a dose of 0.5 to $0.6 \mathrm{mg} / \mathrm{kg}$.

Intubation was performed and anesthesia complementation with Sevorane, aiming Bis around 50 as a target.

Measurements of pressure, heart rate, bis, Sat O2, expired $\mathrm{CO} 2$, inspired and expired sevorane; will be collected before intubation, Post intubation, and surgery sequence until after extubation, as well as any complications. 
Furthermore, data on pain after surgery completion and extubation, intraoperative hydration, blood loss, and diuresis during surgery will be recorded.

\section{- Intraoperative:}

Sufentanil 10ug plus what was left of clonidine to complete 300ug.

\section{- Postoperative:}

Complete the $50 \mathrm{ug} / \mathrm{Kg}$ of morphine.

All rescues via epidural catheter

Observation: Intraoperatively, when analgesic rescue was needed, the prescribed solution was defined with $10 \mu \mathrm{g}$ of sufentanil and $100 \mu \mathrm{g}$ of clonidine diluted with $0.9 \%$ saline solution in $10 \mathrm{~mL}$; applied via epidural twice if necessary. In the immediate postoperative period, if necessary, the application of analgesic rescue for moderate to severe pain; epidural morphine up to $50 \mathrm{ug} / \mathrm{kg}$.

\section{INCLUSION AND EXCLUSION CRITERIA}

\section{Inclusion}

Patients who meet the following criteria will be included in the study:

* Individuals with an indication for upper floor surgery (pancreatectomy, nephrectomy, aortic aneurysm, thoracotomy, and hepatectomy, or L3/L4 for lower abdominal floor surgery (cystectomy, rectosigmoidectomy, prostatectomy).

* Individuals who sign the Informed Consent Form;

* Participation in all stages of the research.

\section{Exclusion}

This study will use the following exclusion criteria:

* Individuals who do not indicate the use of clonidine, sufentanil, and/or morphine.

* Use of obesogenic and/or anticoagulant medications;

* Severe psychiatric disorders;

* Pregnancy during the study period

* Loss of follow-up

\section{OUTCOMES}

\section{Primary Outcome}

Individuals are expected to produce neuraxial sympathectomy, leading to excellent analgesic status both intraoperatively (clonidine and sufentanil) and postoperatively (clonidine and morphine).

\section{Secondary Outcome}

An anesthetic plan is expected to reach around 50 in the BIS and targeted perfusion parameters with around Lactate below 2, mixed venous saturation above $80 \%$, arterial and venous $\mathrm{CO}_{2}$ difference less than six, and hemoglobin above $10 \mathrm{~g} / \mathrm{dL}$.

\section{STATISTICAL ANALYSIS}

For data analysis, a database will be built in a Microsoft Excel spreadsheet which will be exported to the statistical program Minitab 18ß. Common descriptive statistical analysis and the Anderson-Darling normality test will be performed for all non-dichotomous numerical variables, such as BMI, age, and gender. 
According to the results of the Normality Test, the Kruskal-Wallis non-parametric test will be applied, adopting $\alpha$ level $<0.05$ with statistically significant difference $(95 \% \mathrm{CI})$, or the Chi-square parametric test, adopting $\alpha$ level $>0.05$ with statistically significant difference $(95 \% \mathrm{CI})$. Due to the presence of age and gender continuous predictors, linear regression analysis will be applied, adopting $\alpha$ level $<0.05$ with significant influence $(95 \% \mathrm{CI})$.

\section{ORGANIZATION AND INFRASTRUCTURE}

The study will be single-center and will be carried out at the Base Hospital of São José do Rio Preto, São Paulo, Brazil.

\section{FUNDING}

Own financing.

\section{SCHEDULE}

This study will last for six months.

\section{REFERENCES}

1. Womble TN, Comadoll SM, Dugan AJ, Davenport DL, Ali SZ, Srinath A, Matuszewski PE, Aneja A. Is supplemental regional anesthesia associated with more complications and readmissions after ankle fracture surgery in the inpatient and outpatient setting? Foot Ankle Surg. 2021 Jul;27(5):581-587. doi: 10.1016/j.fas.2020.07.015. Epub 2020 Aug 14. PMID: 32917527.

2. Lumas S, Hsiang W, Akhtar S, Ochoa Chaar CI. Regional Anesthesia is Underutilized for Carotid Endarterectomy Despite Improved Perioperative Outcomes Compared with General Anesthesia. Ann Vasc Surg. 2021 May;73:336-343. doi: 10.1016/j.avsg.2020.11.035. Epub 2020 Dec 26. PMID: 33373769.

3. Turner EHG, Whalen CJ, Beilfuss MA, Hetzel SJ, Schroeder KM, Spiker AM. Neuraxial Anesthesia Is Associated With Decreased Pain Scores and Post-Anesthesia Care Unit Opioid Requirement Compared With General Anesthesia in Hip Arthroscopy. Arthroscopy. 2021 Jan;37(1):139-146. doi: 10.1016/j.arthro.2020.08.032. Epub 2020 Nov 18. PMID: 33220466.

4. Rockemann MG, Seeling W, Duschek S, Reinelt H, Steffen P, Georgieff M. Epidural bolus clonidine/morphine versus epidural patient-controlled bupivacaine/sufentanil: quality of postoperative analgesia and cost-identification analysis. Anesth Analg. 1997 Oct;85(4):864-9. doi: 10.1097/00000539199710000-00028. PMID: 9322471.

5. Akter N, Ratnayake B, Joh DB, Chan SJ, Bonner E, Pandanaboyana S. Postoperative Pain Relief after Pancreatic Resection: Systematic Review and Meta-Analysis of Analgesic Modalities. World J Surg. 2021 Oct;45(10):3165-3173. doi: 10.1007/s00268-021-06217-x. Epub 2021 Jun 29. PMID: 34185150; PMCID: PMC8408074. 